CORPUS $\begin{aligned} & \text { Corpus } \\ & \mathbf{5 | 2 0 0 6} \\ & \text { Corpus et stylistique }\end{aligned}$

\title{
Vers une stylistique des imaginaires langagiers
}

Julien Piat

\section{(2) OpenEdition}

Journals

Édition électronique

URL : http://journals.openedition.org/corpus/441

DOI : $10.4000 /$ corpus.441

ISSN : 1765-3126

\section{Éditeur}

Bases ; corpus et langage - UMR 6039

\section{Édition imprimée}

Date de publication : 1 décembre 2006

Pagination : 113-141

ISSN : 1638-9808

\section{Référence électronique}

Julien Piat, "Vers une stylistique des imaginaires langagiers », Corpus [En ligne], 5 | 2006, mis en ligne le 29 juin 2007, consulté le 08 septembre 2020. URL : http://journals.openedition.org/corpus/441 ; DOI : https://doi.org/10.4000/corpus.441

Ce document a été généré automatiquement le 8 septembre 2020

(c) Tous droits réservés 


\title{
Vers une stylistique des imaginaires langagiers
}

\author{
Julien Piat
}

Quel corpus pour quelle stylistique?

1 Tenter d'évaluer la pertinence des corpus pour l'analyse stylistique suppose qu'on ait résolu la question suivante: qu'entendre par «analyse stylistique»? Or, les modèles d'analyse stylistique sont multiples, et c'est bien ce qui pose problème à certains - la stylistique, ce n'est rien d'autre que de l'analyse littéraire, disent-ils, qu'on essaie de masquer derrière un jargon trop technique. La stylistique comme discipline scientifique, malgré son histoire et ses acquis aujourd'hui indéniables, manque encore de légitimité ${ }^{1}$.

2 Née sur les ruines de la rhétorique, à la fin du xix ${ }^{\mathrm{e}}$ siècle, la stylistique est la fille de son temps : issue de la philologie allemande autant que de la linguistique psychologique, elle a pour principe un double héritage descriptif et subjectiviste ${ }^{2}$, qui à la fois explique et problématise le statut des corpus sur lesquels elle s'appuie.

3 La stylistique essentiellement linguistique de Charles Bally est tout entière orientée vers l'analyse de la subjectivité dans la langue. La réflexion de Bally se veut générale; la langue littéraire s'en trouve donc exclue qui, s'éloignant par principe de l'usage standard, est une langue de spécialité. En même temps le style est ramené du côté d'un usage individuel de la langue, et le corpus littéraire ne peut alors être laissé de côté, bien qu'il vise moins la communication des sentiments (objet de la stylistique qui intéresse Bally) que la recherche esthétique (objet de l'analyse de style). En d'autres termes, le dernier de ces usages relève d'une utilisation consciente des ressources de la langue, le premier, non. Mais "s'il existe une "stylistique individuelle", cela suppose nécessairement que la "langue commune" contient en germe des procédés qui autorisent une relative créativité linguistique" (voir Gouvard, 2005: 15-17). La démarche problématique de Bally pointe aussi, à travers ses hésitations mêmes, vers l'existence d'une intention, que ce soit dans l'usage standard ou dans l'œuvre littéraire. Et la notion sera appelée à bien des développements dans le domaine stylistique, mais au prix d'une réduction quasi exclusive au corpus littéraire. 
4 De fait, cette catégorie d'intention fonde ce qu'on pourrait appeler la deuxième stylistique, dont la visée herméneutique cherche à s'appuyer sur des formes langagières. La voie est ouverte par Spitzer, dans ses Études de style de 1928. Son idée d'« étymon spirituel », par-delà ce qu'elle recoupe, illustre les problèmes qui seront toujours ceux de cette pratique stylistique: le corpus définit-il la base même de la construction $\mathrm{du}$ sens ou est-il constitué a posteriori par le stylisticien, afin d'illustrer une thèse préétablie? Si la démarche spitzerienne peut être taxée d'«hypocrite", c'est bien qu'elle ne pose pas assez clairement la question de son corpus. Une difficulté de la même espèce se retrouve dans la stylistique structurale inaugurée par Riffaterre : il ne s'agit plus de mettre au jour ce qui fonde un « étymon spirituel » ou une visée (vision ?) particulière, mais de déterminer ce qui fait sens par rapport - par écart ? - au corpus considéré - le « texte » clos.

De telles approches démontrent que le corpus n'est jamais que ce que l'on veut en faire - il est pris au cœur d'enjeux et de méthode qui le dépassent. Mais elles demeurent peut-être trop exclusives les unes des autres. Bordas (2005) trace alors une voie féconde, avec pour horizon « un travail qui inviterait [...] à réfléchir sur l'imaginaire linguistique d'un auteur, ses évolutions, ses permanences, son esthétique en somme». Ce qui signifie «militer pour une stylistique qui partirait de la langue pour aller vers le style, perçu dans toute la vérité de sa réalité plurielle $»^{3}(30)$. Un tel programme implique une perspective décentrée : le fétichisme du texte (et donc, du corpus) devrait être dépassé grâce à une recontextualisation large. La stylistique se fait alors moins analyse d'un texte ou d'un auteur que d'un moment. Et le corpus sur lequel elle se fonde nécessairement - parce qu'elle s'intéresse aux formes langagières mises en œuvre dans les textes littéraires - se transforme ainsi en étalon.

6 Sans tout rejeter des précédents développements de la stylistique - la récurrence et la fréquence de certaines formes, au sein du corpus, définissent ce qui est significatif (voir Molino, 1994) - on se propose de dégager, à partir de formes récurrentes, des patrons stylistiques ${ }^{4}$. Mais parce que le statistique ne saurait définir ni le sens ni la spécificité esthétique du texte littéraire, on doit demeurer attentif aux deux types existants de "prédicats stylistiques»: les prédicats caractérisants, "répondant à la question comment? ", et les prédicats déterminatifs, « répondant aux questions de qui ? d'où ? de quand?» (Vouilloux, 2005 : 325). Manière de redire que l'attention du stylisticien doit se porter sur le fonctionnement interne aussi bien qu'externe de son corpus. On comprend ainsi que l'analyse stylistique s'intéresse non seulement aux effets dont le texte est à l'origine mais encore à leurs conditions de possibilité.

7 À l'horizon de cette étude se situe donc une réflexion sur le statut même de la stylistique et sur celui de la langue littéraire, mais sans " déshistoriciser la question » pour reprendre les termes de Maingueneau (2004: 156). Voyons alors comment un corpus peut être constitué par les romans de Samuel Beckett, Robert Pinget et Claude Simon parus dans les années $1950^{5}$, et comment ce même corpus peut illustrer une telle démarche - définir, à partir d'une périodisation de l'histoire littéraire et à travers la récurrence de patrons stylistiques, un moment des imaginaires langagiers.

1. Effet de lecture et histoire littéraire

8 Un corpus d'étude digne de ce nom doit répondre - ne serait-ce qu'implicitement - à la question suivante : pourquoi cette réunion de textes plutôt qu'une autre? On ne peut en effet parler de corpus que dans le cadre d'une problématique. Un corpus, s'il mérite son nom, doit être l'objet d'une construction intellectuelle, qui peut soit lui pré-exister - et 
dans ce cas-là, l'analyse stylistique sera déductive, attachée à repérer des formes validant une hypothèse de départ - soit être constituée à partir d'un ensemble de textes - de manière inductive: des formes langagières acquièrent une certaine représentativité et font signe vers un "principe d'harmonie» (Cahné, 1994: 69) régissant l'ensemble du texte ou de l'œuvre envisagé(e). Cependant, la dichotomie n'est sans doute pas tenable, et l'on ne doit céder à l'illusion ni de la déduction, ni de l'induction. Le corpus pertinent pour l'analyse stylistique ne peut se construire que dans la complémentarité des deux démarches.

D'une part, toutes deux postulent une nécessaire unification: soit il s'agit de «se donner les bases empiriques nécessaires pour répondre à un questionnement théorique ou étayer une hypothèse structurale " (Mellet, $2002: 7)^{6}$, soit il s'agit, inversement, de remonter à partir du corpus vers un principe unificateur'. D'autre part, les effets de lecture peuvent rencontrer des cadres discursifs ${ }^{8}$ contextuels plus larges, ou vice-versa.

Plus largement, la question qui se pose ici est elle-même double: elle concerne les délimitations interne (sur quoi s'appuie-t-on pour réunir des textes?) et externe (sur quoi s'appuie-t-on pour exclure d'autres textes?) du corpus.

\subsection{Un effet de lecture}

11 On partira d'une intuition de lecture : avec Beckett, Pinget et Simon, on est face à un corpus que l'on peut qualifier de « difficile» (Chambers, 1982). Mais d'où provient cette difficulté ? Comment en rendre compte?

En faisant référence, sans aucun doute, à un type d'écriture romanesque - les textes sont explicitement désignés comme "romans » sur la page de couverture -, qui ne correspond pas aux codes traditionnels. On peut songer à la difficulté - l'impossibilité ? - de résumer l'«histoire» de L'Innommable ou de Mahu, au dérèglement de la chronologie dans La Route des Flandres ou à la structure même de Molloy, composé de deux parties qui, malgré bon nombre d'échos, demeurent difficilement conciliables. Mais ce qui vaut pour l'un des textes ne vaut pas nécessairement pour les autres (on peut, par exemple, résumer l'intrigue de La Route des Flandres).

Il faut alors formuler une hypothèse décalée: si les outils narratologiques ou rhétoriques ${ }^{9}$ ne peuvent rendre compte ni des effets de lecture - question principielle pour l'analyse de style - ni des dysfonctionnements perçus, c'est parce qu'ils demeurent au niveau du sens. Contre une stylistique trop abstraite du roman, fondée sur ces "formes générales» que sont «les genres littéraires ou encore [1]es types discursifs » (Gouvard, 2005b : 19), on veut jouer, d'abord ${ }^{10}$, la carte de la matérialité textuelle : ce qui fonde ce regroupement de textes en un corpus, c'est, empiriquement, un traitement expérimental de la langue.

14 Ce travail de la langue est à l'origine d'une grande opacité référentielle : certaines formes, essentiellement syntaxiques, brouillent la construction de l'univers romanesque - soit parce qu'elles vont contre l'usage attendu de la langue et de ses structures, soit parce qu'elles jouent avec les mouvements de prédication sur lesquels repose exclusivement le monde de la fiction. Pour illustrer ces problèmes, sans encore entrer dans le détail, on peut considérer les trois extraits suivants :

[1] Quand tout se taira, quand tout s'arrêtera, c'est que les mots auront été dits, ceux qu'il importait de dire, on n'aura pas besoin de savoir lesquels, on ne pourra pas savoir lesquels, ils seront là quelque part, dans le tas, dans le flot, pas forcément les derniers, il faut qu'ils soient avalisés par qui de droit, ça prend du temps, il est loin, qui de droit, c'est le maître, on lui apporte le procès-verbal, tous les, il connaît 
les mots qui comptent, c'est lui qui les a choisis, pendant qu'on va vers lui, pendant qu'il cherche, pendant qu'on revient vers nous, avec le verdict, les mots continuent, les mauvais, les faux, jusqu'à ce que l'ordre arrive, de tout arrêter, ou de tout continuer, non, inutile, tout continuera tout seul, jusqu'à ce que l'ordre arrive, de tout arrêter. (Beckett, $1953: 138$ )

[2] Petit à petit avec les années le chahut s'est calmé, on ne m'a plus demandé si je dormais feignant, on m'a laissé sortir mon bras des couvertures et attendre qu'on me réveille peut-être. (Pinget, [1952] 1956 : 11)

[3] Comme celle (l'expression) qui pouvait se lire sur son visage (à Montès) quand je lui parlai de Cécile, lui demandai s'il l'avait revue. (Simon, 1957 : 216) phrase d'insertions, de répétitions, de ruptures syntaxiques et de décrochements énonciatifs; Pinget ne respecte pas les règles de transposition de l'interrogation indirecte (feignant devrait disparaître); Simon, après une phrase longue, recourt à l'anaphore pronominale alors même que le référent n'est plus accessible - d'où son nécessaire rappel, entre parenthèses.

Le choix d'extraits peut paraitre arbitraire. La démarche est pourtant fondamentale dans l'analyse stylistique des textes. Le mouvement de réduction, nécessaire à toute constitution de corpus, y trouve une autre réalisation. Les variations dans «l'empan du regard"porté sur tout corpus imposent des réajustements (Mellet, 2002: 6). La réduction à un ensemble de textes, puis de cet ensemble de textes à un ensemble d'extraits s'explique par le choix du significatif. Si le corpus général est fondé sur une parenté d'effets de lecture généraux, l'analyse stylistique ne peut s'en tenir à ce seul niveau : elle est prise par nature dans un va-et-vient entre le général et le local ${ }^{11}$. Qui plus est, le local peut renforcer l'impression d'ensemble, comme en [2] : la construction agrammaticale repérable ici a beau être un hapax dans Mahu, elle fonctionne comme une marque de lecture difficile. Au contraire, chez Simon, la nécessité de repréciser le fonctionnement de l'anaphore pronominale est fréquente, et constitue un stylème facilement repérable. Quant à Beckett, à côté de phrases aussi amplifiées que [1], on trouve aussi des passages particulièrement morcelés, comme [4], toujours dans L'Innommable :

[4] Je vais avoir de la compagnie. Pour commencer. Quelques pantins. Je les supprimerai par la suite. Si je peux. (Beckett, $1953: 8$ )

On pressent ainsi que l'étude stylistique dépend du choix d'un niveau d'analyse large, permettant, en première approximation, d'instaurer une série de repères. Dès lors que ce principe d'unité est reconnu, un corpus de base est fondé; les divergences repérables au sein d'un ensemble réduit par rapport à ce dernier (comme c'est le cas de nos extraits) permettent de préciser l'analyse, mais pas de remettre en cause son hypothèse directrice : il s'agit bien à la fois de " donner à voir cette réalité trop vaste pour être embrassée dans sa totalité » (Mellet, 2002: 7) qu'est, ici, l'expérimentation langagière, et de fonder la réflexion à partir du principe général d'un effet de lecture difficile.

\subsection{Une contextualisation nécessaire}

Mais en se plaçant du côté de la réception des textes, on ne peut ignorer que le corpus d'étude est cohérent d'un autre point de vue : les romans de Beckett, Pinget et Simon qui retiennent notre attention s'inscrivent dans un moment particulier de l'histoire littéraire, les années 1950, caractérisées, notamment, par une volonté de renouvellement du genre romanesque. Que le Nouveau Roman soit une simple 
étiquette, dont l'à-propos puisse être remis en cause, importe peu dans la perspective stylistique : on dispose là d'un cadre socio-historico-littéraire, décisif pour asseoir la constitution d'un corpus. Il s'agit essentiellement d'un principe régulateur, qui empêche d'araser les perspectives: si la lecture de Proust est elle aussi difficile, les enjeux ne sont pas les mêmes. Mais cela, ce ne sont pas les formes langagières qui le disent a priori ${ }^{12}$, c'est le discours critique, entendu au sens large ${ }^{13}$.

Beckett, Simon et Pinget ne sont pas les seuls auteurs du Nouveau Roman - et qui plus est, ils ne sont pas tous systématiquement répertoriés comme tels ${ }^{14}$. Quant à la lecture des autres nouveaux romanciers, elle est ressentie comme tout aussi difficile. La contextualisation doit alors prendre le relais de l'effet de lecture : ce sont des principes exogènes qui vont justifier l'exclusion de Robbe-Grillet, Sarraute ou Duras - par exemple. Par-delà les effets de réception, on doit s'intéresser à des traits propres au champ littéraire des années 1950 : le regroupement de Beckett, Pinget et Simon passe par une maison d'édition commune et par des stratégies littéraires proches.

Il convient d'abord de se défaire de l'étiquette "Nouveau Roman » : elle s'est révélée utile ${ }^{15}$, mais elle n'est plus suffisante. Une autre catégorie semble plus efficace, celle d'« École de Minuit ", que l'on trouve parfois dans la critique littéraire : Beckett, Pinget et Simon sont des auteurs « Minuit $»^{16}$. À partir du moment où ils entrent chez Minuit 1951 pour Beckett, 1956 pour Pinget, 1957 pour Simon -, ils n'en repartiront pas ${ }^{17}$. Ce qui est encore le cas de Robbe-Grillet, mais pas celui de Sarraute - dont seul Tropismes rejoindra, dans une nouvelle édition ${ }^{18}$, le catalogue Minuit, en 1957 -, Butor, ou Duras. Quand on ajoute à cela la position des Éditions de Minuit dans le champ littéraire - du côté du pôle de "production restreinte " défini pour Bourdieu par l'indifférence aux chiffres de vente (voire par la revendication de tirages faibles ${ }^{19}$ ), et par la volonté de rechercher le plus haut profit symbolique (culturel) ([1992] 1998: 356) - on peut alors comprendre que l'effet de lecture produit par une langue expérimentale entre en parfaite résonance avec ce cadre externe.

21 Mais tel n'est pas encore le corpus d'étude : il reste à exclure Robbe-Grillet, pourtant considéré comme le pape du Nouveau Roman, en vertu de son rôle de directeur littéraire des Éditions de Minuit - fonction qu'il occupe de 1955 à 1985 -, et qui le conduit à repérer Pinget ou Simon, mais grâce encore aux écrits théoriques qu'il fait paraître dès les années 1950, avant de les réunir en 1963 dans Pour un nouveau roman. Avec Sarraute et son Ère du soupçon (paru en 1956), Robbe-Grillet est le théoricien majeur du Nouveau Roman. Un rôle que ni Beckett, ni Pinget, ni Simon n'assumeront ${ }^{20}$. Chez eux - et cela intéresse alors directement l'analyse stylistique -, la refondation du roman passe par ce qu'on peut appeler des manifestes pratiques. Une telle fonction expliquerait que leurs textes soient le lieu d'un travail sur la langue qui n'apparait pas chez Robbe-Grillet.

Le corpus d'étude se réduit donc à un ensemble « Nouveau Roman - École de Minuit manifestes pratiques ", susceptible de cadrer avec les effets de lecture intuitifs. Mais dès lors, un renversement a lieu : le cadre externe, parce qu'il est étayé sur des acquis scientifiques, semble devenir le fondement même de l'entreprise stylistique. C'est à partir de cette assise, moins susceptible d'être invalidée que de simples impressions de lecture, que l'on va pouvoir à nouveau considérer le grain des textes.

Deux critères permettent donc de délimiter un corpus polygraphique: un critère interne - la difficulté de ces textes -, d'ordre esthétique, appuyé sur des faits linguistiques, et un critère externe - ces textes sont des manifestes pratiques et ont 
tous été publiés aux Éditions de Minuit. Les intuitions de lecture enclenchent l'analyse ; une relecture du contexte sélectionne le regroupement le plus pertinent. Car il ne peut en aller que de degrés de pertinence: « un corpus ne peut être clos et exhaustif que dans le cadre d'une monographie "; mais un corpus monographique "sera étudié en tant que tel, sans prétendre à être représentatif d'autre chose que de lui-même ni à ouvrir sur aucune forme de généralisation ou modélisation» (Mellet, 2002:6). Or, tenter de rendre compte des imaginaires langagiers d'un moment donné - à travers la langue littéraire qu'ils modèlent - suppose une pratique nécessairement comparative.

2. Des patrons stylistiques

Le corpus auquel on aboutit témoigne d'un moment caractérisé par une surautonomisation du champ littéraire. On peut en effet réévaluer les intuitions de Bourdieu, portant elles sur l'autonomisation du même champ dans le second XIX siècle. Le processus se serait caractérisé par une attention plus nette portée au style - c'est-àdire à la langue elle-même. Mais ce qui échappe à Bourdieu, c'est qu'à partir de ce moment, la langue littéraire va présenter des formes qui lui seront propres ${ }^{21}$. L'exclusion progressive des exemples littéraires dans les grammaires scolaires prouve que l'écrivain n'est plus apte à garantir l'usage commun de la langue : le sien est devenu trop déviant pour servir de modèle. Phrases averbales, utilisation de l'imparfait pour le premier plan narratif, jeux sur l'ordre des mots, discontinuité syntaxique, substantivations massives dans l'écriture artiste, ruptures de la transitivité verbale : ce sont quelques-uns des traits que la langue littéraire française adopte au tournant du siècle.

C'est ce déplacement de la littérarité du côté de la langue, envisagée comme laboratoire, qui va permettre de comprendre comment une diversité formelle et stylistique est susceptible de se maintenir à l'intérieur du corpus, et en même temps d'être dépassée. Par-delà la ressemblance ou la dissemblance stylistique, c'est un même geste, ressortissant à un même imaginaire langagier qui doit être recherché.

2.1. Une stylistique des patrons

En revenant aux textes, il semble bien que les pratiques langagières des uns ne soient pas réductibles à celles des autres. La problématique idiolectale du style resurgit, qui fait remonter une pratique de la langue à l'individualité d'un auteur. Si cette stylistique d'auteurs a rapidement été enterrée (grosso modo avec Riffaterre ${ }^{22}$ ), elle l'a sans doute été trop vite. Le développement, en linguistique, des problématiques énonciatives, dans les années 1970, a eu tôt fait de relancer le débat (voir Philippe, 2005). Or, si l'on souhaite rendre compte de l'état de la langue littéraire à un moment donné, il faut sans doute se rapprocher du pan de la stylistique qui, dans le sillage de Bally (Gouvard, 2005), a pu se définir comme une "linguistique des idiolectes littéraires» (Philippe, 2006). Mais sans perdre de vue que cette pratique visait à rendre compte, en s'appuyant sur les divers idiolectes littéraires, du sociolecte qu'est, en fin de compte, la langue littéraire ${ }^{23}$.

La comparaison prend alors tout son sens : sans gommer les différences, elle parvient à un recul suffisant pour ordonner et classer des phénomènes langagiers. Pour sortir de l'aporie, on doit reconnaître que le style se situe au carrefour d'une pratique singulière et d'une pratique collective de la langue. Or, dans l'état du champ littéraire considéré ici, cette pratique collective est moins définie en termes sociaux que sémiotiques littéraires. En dégageant un ensemble de patrons stylistiques, on parviendra alors à subsumer les pratiques individuelles, en même temps qu'à les définir positivement ou 
négativement - un auteur peut travailler avec un patron ou contre lui. L'abstraction semble donc le risque à prendre dans l'analyse stylistique, mais comme un préalable. Les modèles stylistiques ainsi dégagés serviront de comparants pour l'ensemble des textes d'un corpus. Ils introduiront aussi une dynamique dans la démarche stylistique : en synchronie, c'est le corpus de base qui pourra servir d'étalon pour un corpus élargi.

On dégage de tels patrons en s'appuyant sur la récurrence et la fréquence de certains phénomènes, ces deux caractéristiques relevant du domaine de la réception, indépendamment de toute valeur intentionnelle. C'est donc des attendus langagiers du lecteur qu'il faut tenir compte. Or, les effets de lecture difficile créés par notre corpus semblent tous naître de coups portés à la phrase - définie comme unité typographique et prédicative. On croit en effet que l'existence d'une conscience phrastique chez le lecteur, appuyée sur une phrase idéale, est un critère de premier choix pour évaluer le style $^{24}$. C'est parce que Beckett, Pinget et Simon travaillent tous trois aux limites de la phrase, tant sur les plans typographique et syntaxique que logique, que l'on peut dégager l'ensemble des patrons stylistiques définissant une langue littéraire expérimentale.

2.2. Un double patron : maximalisme et minimalisme stylistiques

[1] et [4] peuvent a priori constituer les deux pôles du champ d'investigation. Dans [1], la phrase est nettement amplifiée; dans [4], elle est démembrée. Si l'on parcourt les autres romans du corpus, on trouve la même double tendance chez Pinget, où l'on peut comparer :

[5] Les émigrants, massés sur tous les ponts, voyaient s'approcher cette terre réelle, vivante hors de leur esprit tout à coup, celle de l'attente profondément mûrie et peu à peu légendaire comme un portrait fait en dormant qu'on trouve au réveil sur le mur, réelle avec ses montagnes et sa ville, elle est crénelée de remparts, fortifiée contre mille invasions impuissantes, ouverte, béante, tous ses oriflammes au vent, hurlante, précipitée des nuages comme une pluie torrentielle, elle pousse devant elle ses milliards de fleurs subites, et du fond des vieilles querelles pourrissantes se détache à leur rencontre, car sa parole est droite et toutes ses œuvres s'accomplissent dans la fidélité, il rassemble comme en un monceau les eaux de la mer, il réduit à néant les desseins des nations, il met obstacle aux pensées des peuples et subsistent les pensées de son cœur de générations en générations. ([1953] 1971: 79)

[6] Je ne voulais pas, je voulais. T'écrire. ([1959] $1981: 27)$

On trouve principalement chez Simon des phrases qui courent sur plusieurs pages ${ }^{25}$, mais on peut aussi relever des occurrences comme :

[7] Mais même alors il ne bougea pas. Plus tard il me raconta qu'il avait senti se produire en lui quelque chose de bizarre. (1957: 98)

[7] ne peut cependant être confondu avec [4] ou [6] : les attendus phrastiques n'y sont pas battus en brèche, comme, apparemment, ce peut être le cas chez Pinget :

[8] Un mardi nous recevions la reine de Dualie en visite officielle. Des questions de politique avaient décidé Baga à ce geste très onéreux. On ne pouvait guère offrir plus qu'un pique-nique, mais il devait être royal. La reine Conegrund était réputée pour son appétit d'ogresse et pour son goût de la parade. (1958:131)

et chez Beckett :

[9] Il est minuit. La pluie fouette les vitres. Je suis calme. Tout dort. Je me lève cependant et vais à mon bureau. Je n'ai pas sommeil. Ma lampe m'éclaire d'une lumière ferme et douce. Je l'ai réglée. Elle me durera jusqu'au jour. ([1951] 1999: 125) 
L'extension de la phrase est réduite, et en cas de prédications multiples, la structure reste claire : un cas de subordination en [7], des faits de coordination en [8] et [9]. Pourtant, si chaque phrase correspond individuellement au canon, leur succession, c'est-à-dire leur accumulation au sein d'un texte, semble poser problème : la linéarité romanesque repose sur un principe de causalité, que garantit d'abord la cohésion textuelle, et qui se trouve alors remis en question. L'absence de lien explicitement marqué entre ces phrases provoque une sorte de discontinuité discursive. L'effet de lecture relève bien d'une contradiction entre les cadres généraux (génériques) et les faits locaux (langagiers).

La brièveté des phrases définit un patron stylistique minimaliste, actualisable sous différentes formes. Dans [4] ou [6], l'usage marqué ne provient pas de l'accumulation de phrases minimales mais de la répartition problématique de l'information. Si la phrase idéale est associée à une unité prédicative, ces occurrences diffractent la prédication en plusieurs unités phrastiques - ce qui est marqué en [4] par l'autonomisation de propositions en phrases, en [6] par la non-coïncidence entre découpage typographique et réalisation de la valence verbale.

Inversement, [1] et [5] présentent des phrases particuliè-rement amplifiées - jusqu'à l'explosion, chez Simon. Pour synthétiser l'ensemble de ces pratiques, on peut proposer d'identifier un patron maximaliste appuyé sur le recours aux insertions ${ }^{26}$, qu'il s'agisse d'appositions en [5], de subordonnées en [1], ou, chez Simon, de syntagmes participiaux comme en [10]:

[10] [...] Georges se rappelant avoir d'abord été frappé par l'ombre [...] Georges parvenant alors seulement à comprendre ce que criait la voix [...] entendant sa propre voix sortir [...] et Georges hurlant alors aussi [...] ([1960] 1998 : 101-102)

La tendance à l'autonomisation de ces segments au sein de la phrase, proportionnellement à leur longueur, est à l'origine des difficultés de lecture. À travers leur accumulation, ces segments changent de statut: d'a priori secondaires dans la construction de l'information et de la référence, ils acquièrent une importance dans l'économie de la phrase ${ }^{27}$. Qui plus est, les jeux de prédication qu'ils mettent en œuvre peuvent renforcer cette impression, et notamment la multiplication des insertions propositionnelles - notamment chez Beckett -, qui répète le modèle physique et topographique de la phrase. C'est encore de cette manière que peut s'expliquer, chez Simon, la perte de saillance des référents au sein des chaînes d'anaphore (voir [3]).

2.3. Un patron génétique

$37 \mathrm{Au}$ sein de ces configurations prédicatives problématiques, il en est une, particulièrement fréquente chez Beckett et Pinget, fondée sur la multiplication des commentaires méta-énonciatifs : quand

le dire se représente comme n'allant plus «de soi », le signe, au lieu d'y remplir, transparent, dans l'effacement de soi, sa fonction médiatrice, s'interpose comme réel, présence, corps - objet rencontré dans le trajet du dire et qui s'y impose comme objet de celui-ci -; l'énonciation de ce signe, au lieu de s'accomplir "simplement», dans l'oubli qui accompagne les évidences inquestionnées, se redouble d'un commentaire d'elle-même ${ }^{28}$.

38 Mais le phénomène n'est pas réductible à une seule construction syntaxique. Il s'affiche au niveau intrapropositionnel :

[11] [...] à chaque moment naissaient et disparaissaient des espèces de petits flocons qui n'étaient en réalité que les gardiens allant et venant, mêlés peut-être j'allais dire 
aux prisonniers, car vue de cette distance la cape n'avait plus de rayures ni même l'aspect d'une cape [...]. (Beckett, $1951: 173)^{29}$

[11'] Monsieur Levert se rendait à ce qu'on appelle le village [...] (Pinget, [1959] 1981 : 53)

aussi bien qu'en position transpropositionnelle intraphrastique :

[12] Mon délabrement physiologique parlerait en faveur de cette hypothèse, j'avais peut-être laissé ma jambe dans l'océan Pacifique, que dis-je peut-être, je l'y avais laissée, au large de Sumatra, aux jungles rouges de rafflésie puant la charogne, non, ça c'est l'océan Indien, quelle encyclopédie, enfin par là. (Beckett, 1953 : 51)

[12'] [...] il faut manger pour n'avoir pas le mal de mer, j'ignore si c'est vrai en tout cas ça tombe pile [...] (Pinget, [1953] $1971:$ :42-43)

Enfin, on peut trouver la trace d'une modalité méta-énonciative dans des configurations transphrastiques, comme ici :

[13] Je regrette de ne pas pouvoir indiquer plus clairement de quelle manière ce résultat fut obtenu. Ça aurait fait un beau morceau. Mais ce n'est pas arrivé à ce point de mon récit que je vais me lancer dans la littérature. (Beckett, [1951] 1999: 206)

[13'] Les putains du pays demandent une roupie pour deux roupettes. Assez drôle, ma plaisanterie. (Pinget, $1958: 10$ )

Des difficultés de lecture surgissent de ces structures opacifiantes car elles bloquent, ne serait-ce que momentanément, la construction de la référence fictionnelle. En déplaçant l'attention du dit au dire, les commentaires méta-énonciatifs attirent aussi l'attention sur la production même du discours. C'est là un autre type de patron, "génétique » celui-ci ${ }^{30}$, indépendant, et en même temps complémentaire, des patrons minimaliste et maximaliste ${ }^{31}$.

Si l'on ne retrouve pas les mêmes structures chez Simon, le patron génétique ne s'en trouve pas pour autant invalidé. L'intérêt de la catégorie réside dans sa plasticité relative, ou dans son degré élevé d'adaptation. Le patron est plus proche des effets que des formes elles-mêmes. Dès lors, on peut y faire entrer des mouvements qui, sans être syntaxiquement identiques, ressortissent à une même pragmatique. Chez Simon, les mouvements de correction, de glose, de reformulation multiple en sont des traces fréquentes, structures qui «sont caractérisables, plus ou moins explicitement, comme des formes de retour de l'énonciateur sur son propre dire " (Bikialo, 2003: 297) ${ }^{32}$. L'exemple suivant ${ }^{33}$ est particulièrement frappant :

[14] L'homme-montagne avait depuis longtemps cessé de donner la réplique autrement que sous la forme de ces vagues sons indistincts émis non en guise de réponse mais, pour ainsi dire, de signaux, et encore : signaux non d'attention, d'intérêt pour ce qu'elle racontait, mais en quelque sorte, comme une simple manifestation (le son, le bruit de gorge) d'existence, comme un réflexe déclenché non par les paroles mais par le silence [...], c'est-à-dire lorsque, le flot des paroles cessant, le silence s'instaurant, se prolongeant, s'appesantissant, lui faisait comprendre qu'elle attendait, exigeait, se pensait en droit d'attendre de lui cette marque, cette preuve de sa présence, avant de se remettre à parler (Simon, [1958] $2001: 132$ )

Chacune des séquences soulignées peut être rapportée à une structure de glose, et ramenée, de ce fait, à un avatar du patron génétique global. En effet, "gloser, à l'intérieur d'un discours, c'est expliquer par une autre la parole lâchée " (Douay et Steuckardt, $2005: 5$ ). En d'autres termes, c'est bien faire retour sur la production de l'énoncé. 

corpus sans présager de son unicité. Si de tels outils rendent compte de manière économique des traits stylistiques les plus manifestes d'un corpus, ils ont la capacité de regrouper des faits linguistiques variés, réunis par la proximité de leurs effets. En même temps, la construction de patrons stylistiques est un moyen de pénétrer le texte difficile. Leur émergence au sein de la conscience épistylistique du lecteur permet de programmer en partie la lecture, et de réduire ainsi le risque d'illisible.

3. Stylistique des moments et imaginaires langagiers

Le programme annoncé depuis les premiers pas de cette réflexion implique que si les patrons stylistiques permettent de décrire un corpus avec pertinence, ils doivent aussi être compris comme les piliers méthodologiques d'une relecture de l'histoire littéraire. Ils permettent, croyons-nous, d'analyser l'état de la langue littéraire et, par-delà, les imaginaires langagiers propres à un moment donné. L'étude du corpus initial n'est qu'un préalable : l'entreprise stylistique doit convoquer un nouveau corpus, rouvert, élargi : parce que les patrons sont des outils, ils doivent permettre de comparer les phénomènes expérimentaux présents chez Beckett, Pinget et Simon avec la pratique de leurs contemporains. Or, on sait depuis Bourdieu que la dynamique propre au champ est dominée par des luttes. Les patrons stylistiques, définis grâce aux œuvres les plus expérimentales, pourront certes affiner les comparaisons, voire remettre en cause les classements de l'histoire littéraire externe - il est des auteurs de romans qui jouent avec les limites de la langue et ne sont pas étiquetés comme nouveaux romanciers -, mais aussi mettre en évidence que si certains considèrent la langue littéraire comme laboratoire, d'autres en font un conservatoire (par exemple). C'est bien la question des imaginaires langagiers qui se trouve alors au cœur de la réflexion stylistique.

3.1. Des corpus périphériques?

Une telle méthode suppose un intérêt initial pour les faits les plus saillants, qui sont en effet les seuls à pouvoir positivement fournir des catégories d'analyse rentables. Il faut radicaliser les faits de langue (maximalisme syntaxique vs minimalisme syntaxique) ou les effets de lecture (patron génétique) pour pouvoir disposer de repères efficaces. $\mathrm{Ce}$ n'est qu'ensuite qu'on peut adopter une visée comparative.

Pour être mieux à même de situer les entreprises stylistiques que Giono, Bataille, Sartre, les Hussards ou encore Céline - pour ne citer qu'eux ${ }^{34}$ - mènent dans ces années-là, on s'intéressera d'abord au Hussard sur le toit, roman contemporain de Molloy (1951). D'un côté, un roman de lecture aisée; de l'autre, un roman difficile. Si l'on s'interroge sur la langue de ces textes, on remarquera que le patron génétique est absent chez Giono, ou qu'au minimalisme et au maximalisme syntaxico-stylistiques présents tous deux dans Molloy, répond la neutralité des phrases du Hussard: les insertions n'y sont pas multipliées, la ponctuation jamais détournée de son rôle attendu dans la segmentation phrastique :

[15] Dans la deuxième maison il tomba sur des cadavres un peu moins frais. Ils n'étaient cependant pas pourris, mais secs comme des momies. La dent du chien et le bec des oiseaux les avaient troués de déchirures franchement dentelées, comme mordues et becquetées dans un lard de quatre ans. Ils répandaient malgré tout cette odeur de sirop qui indiquait des cadavres récents. ([1951] 1984 : 272)

Bataille fit paraître Le Bleu du ciel l'année où Minuit publia Le Vent (1957). Soit l'extrait suivant :

[16] Dans cette nuit opaque, je m'étais rendu ivre de lumière ; ainsi, de nouveau, Lazare n'était devant moi qu'un oiseau de mauvais augure, un oiseau sale et 
négligeable. Mes yeux ne se perdaient plus dans les étoiles qui luisaient au-dessus de moi réellement, mais dans le bleu du ciel de midi. Je les fermais pour me perdre dans ce bleu brillant : de gros insectes noirs en surgissaient comme des trombes en bourdonnant. De la même façon que surgirait, le lendemain, à l'heure éclatante du jour, tout d'abord point imperceptible, l'avion qui porterait Dorothea... ([1957] $2004: 175)$

49

$$
\begin{aligned}
& \text { inversé que provoque la série des trois insertions - la phrase ne ressortit pas à un } \\
& \text { patron maximaliste mais semble y tendre. Cette même phrase se caractérise encore par } \\
& \text { l'autonomisation d'une subordonnée comparative. Le mouvement prédicatif est certes } \\
& \text { touché par une telle structure, mais sans que la construction du sens soit vraiment } \\
& \text { perturbée. Le patron minimaliste permet la reconnaissance du fait mais l'effet ne } \\
& \text { correspond pas; la segmentation apparaissait d'ailleurs à l'identique dans la première } \\
& \text { version du texte, celle que Bataille rédigea en } 1935 \text { (2004: 273). } \\
& \text { Dans Féerie pour une autre fois (1952), le patron minimaliste peut être utile pour rendre } \\
& \text { compte du morcellement en phrases - bien que le critère de la ponctuation soit ici } \\
& \text { problématique -, tandis que le patron génétique est sensible dans les faits de } \\
& \text { nomination multiple: } \\
& \text { [17] Et que voilà d'autres artifices! des obus traçants de très loin... des trajectoires } \\
& \text { originales... spirales !... de canons plus loin que Poissy !... l'effet comme s'ils } \\
& \text { cousaient les nuages... les cousaient ensemble... les ourlaient !... en bleu !... mauve! } \\
& \text { jonquille! ([1952] } 1993: 264)
\end{aligned}
$$

51 Le cas Céline pose cependant un autre type de problème. Que fait-on de la production de Céline antérieure au moment dans lequel on se situe ? Des romans comme Mort à crédit (1936) et Guignol's Band (1947) présentent des traits stylistiques proches - sinon identiques - à ceux que l'on trouve dans la trilogie D'un château l'autre, Nord, Rigodon (1957-1969), et que l'on peut ranger, mutatis mutandis, sous le patron minimaliste. Voilà qui, en d'autres termes, soulève, à travers la question d'un corpus d'amont, celle de la réhistoricisation des patrons. Si l'on ignore le travail de Céline vers le minimalisme syntaxique ou le travail de Proust à l'autre bout de l'échelle, ne risque-t-on pas de perdre de vue la dynamique constitutive du fait littéraire? D'un autre côté, à rouvrir ainsi le corpus, où s'arrêter?

52 Le danger majeur est de pratiquer des comparaisons aberrantes, précisément parce qu'elles ne prennent pas en considération les moments de l'imaginaire langagier - celui de Proust, encore proche de Flaubert, n'est pas celui de Simon, précisément parce que Proust et Céline ont déjà écrit au moment où ce dernier entre en littérature; précisément parce que Proust et Céline ont révélé des possibles de la langue littéraire. Il faut avoir conscience des héritages stylistiques, mais ne pas confondre la pratique individuelle de certains - qui, à l'époque de Proust, écrit comme lui ? - avec une pratique commune à un certain nombre d'auteurs contemporains. Ce n'est que de cette convergence que peut naître une réflexion pertinente sur les imaginaires langagiers. Et ce n'est que dans un sens rétrospectif, quand une configuration stylistique s'est cristallisée en patron, que l'on peut considérer un corpus d'amont - et un corpus d'aval, qui se définit avec ou contre ce même patron, et peut, dans ce dernier cas, contribuer à l'émergence d'un nouveau patron, etc.

Les conséquences d'une telle position sont aussi d'ordre épistémologique. La notion d'écart occupe en stylistique une place bien paradoxale. D'un côté, elle est l'une des notions les plus fréquentes - le style évalué comme écart par rapport à la norme 
grammaticale, ou par rapport à la norme du texte considéré. De l'autre, elle est toujours condamnée puisqu'elle renverrait à des entités difficilement définissables - et en premier lieu, cette langue standard, catégorie intuitive, éventuellement statistique mais non théorique.

Or, l'existence de patrons stylistiques donne à cette notion un contenu plus satisfaisant. S'ils indiquent les tendances les plus marquées de la langue littéraire à un moment donné, ils sont susceptibles de devenir des principes de comparaison. Sans vraiment définir de normes d'époque, ils s'appuient sur des récurrences formelles propres - donc des attendus. L'écart serait alors défini par le degré suivant lequel un texte s'inscrit au sein de ces attendus. Face aux configurations les plus marquées, il est des textes qui en présentent des variantes (ce serait Céline face au corpus Beckett-Pinget-Simon), voire qui n'en présentent aucune (les Hussards face au même corpus). On s'éloigne ainsi de toute idée de norme grammaticale ou de norme standard pour non seulement réhistoriciser la question, mais encore la placer au sein des structures qui la modèlent scène englobante du littéraire, scène générique du roman.

En même temps, l'existence de ces patrons préserve de deux écueils : le nivellement des singularités langagières et leur hypostase. En définissant le patron génétique, on a en effet constaté que la pratique de Simon différait de celle de Beckett et Pinget. Cela signifie à la fois que le style de Simon est identifiable comme tel, mais qu'il ne peut être pleinement compris que s'il est recontextualisé dans son horizon langagier. Privilégier la mise au jour de patrons stylistiques, c'est replacer le style dans un moment particulier, témoignant, notamment, de certains imaginaires langagiers.

\subsection{Imaginaires littéraires et imaginaires langagiers}

Les patrons stylistiques impliquent des pratiques concertées de la langue. Dans notre cas, la contextualisation externe, confrontée aux patrons stylistiques employés, permet de comprendre comment et pourquoi les limites de la langue sont explorées - et en premier lieu, comment la notion de phrase se trouve questionnée. À travers la tendance au minimalisme ou au maximalisme - au morcellement ou à l'expansion -, ce sont des problèmes de lecture qui surgissent - depuis la rupture de la linéarité phrastique jusqu'à la hiérarchie des prédications. Mais en considérant ce travail qui vise à faire disparaître la phrase, on prend conscience qu'elle compose une réalité sensible pour Beckett, Pinget et Simon. Tous trois appartiennent à une époque où elle est tenue pour une unité irréductible - parce que signifiante - de la langue : à phrase claire, pensée claire. Toucher à l'unité ainsi valorisée, c'est nécessairement entrer en conflit avec la clarté et la transparence du langage. Penser en termes de générations - notion qui recoupe en partie celle de moment - permet de préciser les choses. La critique de la phrase est, en effet, un fait générationnel : les linguistiques de l'oral, développées dans les années 1950-1960, entérinent le mouvement apparu plus tôt en littérature. Un faisceau de préoccupations communes apparaît donc, qui permet de définir un véritable «moment linguistique » de la littérature française, où les auteurs remettent en question les cadres normatifs de la grammaire, voire écrivent contre eux, tout en proposant d'autres règles de lecture. Le "moment grammatical de la littérature française $»^{35}$, caractérisé par une prise de conscience, commune aux écrivains et aux critiques, des spécificités grammaticales de la langue littéraire, a créé les conditions de possibilité pour une radicalisation portant sur les structures fondamentales de la langue. 
57 Si le mouvement ne concerne pas l'ensemble des écrivains, il occupe le devant du champ littéraire. L'expérimentation langagière apparaît comme un indice supplémentaire de littérarité. Le moment linguistique tend à promouvoir une scène langagière au détriment de la scène romanesque. L'interrogation porte sur les possibles $\mathrm{du}$ romanesque, mais aussi, à travers eux, sur les possibles du langage. La réponse apportée par les manifestes pratiques de notre corpus est la suivante : le roman ne peut rien dire de satisfaisant sur le réel, mais le langage non plus. Cette absence de transparence des signes aux choses est alors configurée par diverses opérations d'expérimentation langagière, que les patrons ont permis d'identifier. Si le patron génétique acquiert une telle fréquence dans les œuvres du corpus, c'est que l'attention est attirée sur le geste d'écriture lui-même, davantage que sur son sens ${ }^{36}$.

Mais il ne s'agit pas de n'importe quelle écriture : les patrons maximaliste, minimaliste et génétique entraînent un gauchissement de la langue littéraire. Le corpus d'étude montre que le style, ce n'est plus la «belle langue ", mais au contraire, ce qui semble aller contre le style même. Beckett l'avait déjà annoncé, avec humour, en 1937 : «La grammaire et le style. Ils sont devenus, me semble-t-il, aussi incongrus qu'un costume de bain victorien ou le flegme d'un vrai gentleman. Un masque. » ([1937] 1984) S'il est encore pris au cœur d'une vision rhétorique du style, dominée par l'elocutio et la question des ornements, Beckett rêve à une conception de la langue littéraire qui ne parviendra à maturité qu'une vingtaine d'années plus tard ${ }^{37}$.

59 Mais cette conception non stylistique n'est finalement qu'un leurre : c'est précisément parce qu'on malmène, tord, distend la langue littéraire que l'on acquiert un style dans les années 1950. Des auteurs comme Bataille, dont on a pourtant vu qu'il jouait à la marge des patrons stylistiques dominants de l'époque, pratiquent eux aussi cette esthétique du gauchissement - parfois par d'autres moyens ${ }^{38}$. Si les patrons stylistiques font signe vers l'imaginaire langagier d'un moment, ils ne résument pas à eux seuls heureusement! - les potentialités de la langue littéraire.

La pertinence du corpus se mesure donc à l'aune de ses résultats. Travailler un ensemble de textes rapprochés par une expérience de lecture sinon identique du moins relativement homogène et par une inscription historico-socio-littéraire commune permet d'aller vers le fait de style local, afin de remonter, par comparaison, vers des patrons stylistiques dont la visée est aussi heuristique. C'est à partir d'eux que peuvent être envisagés le statut et l'état de la langue littéraire, témoin des imaginaires langagiers d'un moment donné. Les patrons stylistiques permettent à la fois de rendre compte de la matérialité langagière des textes d'un corpus (ils s'appuient sur des configurations syntaxiques) et d'aller du côté d'une herméneutique contrôlée : c'est s'intéresser à la manière dont un texte construit du sens - voire du savoir -, davantage qu'aux intentions - prétendues - de l'auteur.

\section{BIBLIOGRAPHIE}

Bibliographie primaire 
Bataille G. ([1957] 2004). Le Bleu du ciel, in Romans et récits. Paris : Gallimard, « La Pléiade ».

Beckett S. ([1951] 1999). Molloy. Paris : Éditions de Minuit, « Double ».

- (1951). Malone meurt. Paris : Éditions de Minuit.

- (1953). L'Innommable. Paris : Éditions de Minuit.

Céline L.-F. ([1952] 1993). Féerie pour une autre fois I, in Romans IV. Paris : Gallimard, « La Pléiade ».

Giono J. ([1951] 1984). Le Hussard sur le toit, in Euvres romanesques complètes IV. Paris : Gallimard, «La Pléiade ».

Pinget R. ([1952] 1956). Mahu ou le matériau. [Paris : Robert Laffont] Paris : Éditions de Minuit.

- ([1953] 1971). Le Renard et la Boussole. [Paris : Gallimard] Paris : Éditions de Minuit.

- (1956). Graal flibuste. Paris : Éditions de Minuit.

- (1958). Baga. Paris : Éditions de Minuit.

- ([1959] 1981). Le Fiston. [Paris : Éditions de Minuit] Lausanne : L’Âge d'homme.

Simon C. (1957). Le Vent. Tentative de restitution d'un retable baroque. Paris : Éditions de Minuit.

- ([1958] 2001). L’Herbe. Paris : Éditions de Minuit, « Double».

- ([1960] 1998). La Route des Flandres. Paris : Éditions de Minuit, « Double ».

Bibliographie secondaire

Authier-Revuz J. (1995). « Ces mots qui ne vont pas de soi ». Boucles réflexives et noncoïncidences du dire. Paris : Larousse.

Beckett S. ([1937] 1984). « German Letter of 1937 », in R. Cohn (éd.) Disjecta. Miscellaneous Writings and a Dramatic Fragment by Samuel Beckett. New York: Grove Press, 51-54 [en allemand] et 170-173 pour la traduction anglaise.

Bikialo S. (2003). Plusieurs mots pour une chose. De la nomination multiple au style de claude Simon. Thèse de doctorat de langue et littérature françaises, Université de Poitiers.

Bordas É. (2005). « Enseigner la stylistique », in J.-M. Gouvard (éd.) De la langue au style. Lyon : Presses universitaires de Lyon, 21-35.

Bourdieu P. ([1992] 1998). Les règles de l'art. Genèse et structure du champ littéraire. Paris : Éditions du Seuil, « Points ».

Bruneau C. (1948-1953). Histoire de la langue française, t. XII et XIII. Paris : Armand Colin.

Cahné P. (1994). « Qu'est-ce que la forme? », in G. Molinié \& P. Cahné (éds.) Qu'est-ce que le style ? Paris : Presses universitaires de France, 63-69.

Chambers R. (1982). « Le texte "difficile” et son lecteur », in L. Dällenbach \& J. Ricardou (éds.) Problèmes actuels de la lecture. Paris : Clancier-Guénaud, 81-93.

Douay F. \& Steuckardt A. (2005). « Avant-propos », in A. Steuckardt \& A. Niklas-Salminen (éds.) Les marqueurs de glose. Aix-en-Provence : Publications de l'Université de Provence, 5-19.

Fontvieille A. (2005). « Figures d'apposition », in J.-M. Gouvard (éd.) De la langue au style. Lyon : Presses universitaires de Lyon, 99-126.

Gardes-Tamine J. (2004). Pour une grammaire de l'écrit. Paris : Belin.

Gouvard J.-M. (éd.) (2005a). De la langue au style. Lyon : Presses universitaires de Lyon. 
Gouvard J.-M. (2005b). « Introduction générale. Linguistique, stylistique et style chez Charles Bally ", in J.-M. Gouvard (éd.) De la langue au style. Lyon : Presses universitaires de Lyon, 5-20.

Karabetian E. (2000). Histoire des stylistiques. Paris : Armand Colin.

Maingueneau D. (2004). Le discours littéraire : paratopie et scène d'énonciation. Paris : Armand Colin.

Maingueneau D. et Philippe G. (2002). « Les conditions d'exercice du discours littéraire », in E. Roulet \& M. Burger (éds.) Les modèles du discours au défi d'un « dialogue romanesque ». Nancy : Presses Universitaires de Nancy, 351-378.

Mellet S. (2002). « Corpus et recherches linguistiques. Introduction », Corpus 1:1-15.

Molino J. (1994). « Pour une théorie sémiologique du style », in G. Molinié \& P. Cahné (éds.) Qu'est-ce que le style ? Paris : Presses universitaires de France, 213-261.

Philippe G. (2002). Sujet, verbe, complément. Le moment grammatical de la littérature française (1890-1940). Paris : Gallimard.

Philippe G. (2005). « Le style est-il une catégorie énonciative ? », in J.-M. Gouvard (éd.) De la langue au style. Lyon : Presses universitaires de Lyon, 145-156.

Philippe G. (2006). « Traitement stylistique et traitement idiolectal des singularités langagières ", Cahiers de praxématique 46.

Philippe G. (à paraître). « Gauchissement syntaxique et grammaire classique : la langue de Bataille dans le cycle Divinus Deus ", in J.-F. Louette \& F. Rouffiat (éds.) Sexe et textes. Lyon : Presses universitaires de Lyon.

Philippe G. \& Piat J. (éds.) (à paraître). La langue littéraire de Gustave Flaubert à Claude Simon. Pour une autre histoire de la littérature française (1850-2000). Paris : Fayard.

Piat J. (2005). «Conscience phrastique et faits de prédication : l'illisible linguistique dans le Nouveau Roman ", in C. Badiou-Monferran, F. Calas, J. Piat \& C. Reggiani (éds.) La langue, le style, le sens. Paris : L'Improviste, 203-214.

Piat J. \& Philippe G. (2006). « La littérature contre la "belle langue” », Le Monde des livres, vendredi 17 février, 2.

Riffaterre M. (1971). Essais de stylistique structurale. Paris : Flammarion.

Simonin A. (1994). Les Éditions de Minuit 1942-1955. Le devoir d'insoumission. Paris : IMEC Éditions.

Vouilloux B. (2005). « Les prédicats stylistiques », in J.-M. Gouvard (éd.) De la langue au style. Lyon : Presses universitaires de Lyon, 319-355.

\section{NOTES}

1.. Voir Bordas (2005 : 21-22) : « devant autant de confusion, les critiques, depuis quelques années, se multiplient à l'encontre de cette "discipline" aux contours flous, qui semble cependant l'enjeu même des études littéraires et leur finalité la plus originale.»

2.. Pour un approfondissement sur ces questions, voir Karabétian (2000).

3.. Bordas (2005: 30). 
4.. Suivant le modèle des « patrons discursifs » que Maingueneau et Philippe définissent comme « représentations imaginaires de plusieurs types de production langagière précis, dont la tradition littéraire a figé les spécificités en une sorte de stéréotypes »; par exemple, « le déchiffrement des textes romanesques repose [...], en tout premier lieu, sur la reconnaissance de tels "patrons" et exige une compétence interprétative spécifique » (2002: 366-367).

5.. La liste de ces romans est donnée dans les références bibliographiques en fin d'article.

6.. Nous soulignons.

7.. Cahné, partant chez Proust de l'expression de la cause, de l'observation des épithètes et de l'analyse des comparaisons, remonte ainsi vers « une forme unique, véritable matrice de son écriture, et qui est son style » : «l'être et le presque rien sont solidaires ». L'opération, qualifiée de " réduction eidétique » (1994:68), offre l'exemple concis d'une pratique inductive de l'analyse stylistique.

8.. On entend « discursif » dans un sens étendu : tout ce qui a rapport au discours considéré, c'est-à-dire aussi à sa valeur littéraire : inscription générique, sociologique, théorique, etc.

9.. Dans sa dimension de dispositio.

10.. On parle bien de la primauté des effets sur les constructions discursives mises en évidence par l'analyse des discours.

11.. La réflexion épistémologique sur la stylistique ne cesse de le répéter, comme le prouve la quasi-totalité des contributions à Gouvard (2005a), et notamment Jaubert (2005).

12.. Du moins de la manière la plus explicite ; on verra que la définition de patrons stylistiques ou syntaxiques permet de rendre compte de la fréquence plus ou moins élevée de certains types de configurations langagières, et que c'est précisément sur ces écarts d'usage que peut être fondée une histoire de la langue littéraire et des imaginaires langagiers.

13.. Discours de l'histoire, de la philosophie ou de la critique littéraire. Ce qui correspond assez à la stylistique évoquée par Bordas (2005 : 30) évoquée plus haut.

14.. La cartographie des nouveaux romanciers est excessivement mouvante : certains y sont toujours rattachés : Robbe-Grillet, Sarraute, Simon, Ricardou, Butor ; on y trouve parfois Pinget, Ollier et Duras ; quant à Beckett, il n'y est que rarement associé.

15.. On peut rappeler les propos suivants de Bourdieu : «Les mots, noms d'écoles ou de groupes, noms propres, n'ont tant d'importance que parce qu'ils font les choses : signes distinctifs, ils produisent l'existence dans un univers où exister, c'est différer, "se faire un nom", un nom propre ou un nom commun (celui d'un groupe). Faux concepts, instruments pratiques de classement qui font les ressemblances et les différences en les nommant, les noms d'écoles ou de groupes [...] sont produits dans la lutte pour la reconnaissance par les artistes eux-mêmes ou leurs critiques attitrés et remplissent la fonction de signes de reconnaissance [...]» $\gg([1992] 1998: 262)$

16.. Beckett avait publié avant 1951 des textes en anglais, ainsi que la traduction de Murphy en français, chez Bordas, en 1947. La trilogie Molloy, Malone meurt, L'Innommable essuie divers refus (notamment chez Gallimard et au Seuil) avant que Georges Lambrichs et Jérôme Lindon ne l'acceptent chez Minuit en 1950. Les premiers romans de Pinget ne sont pas publiés par Minuit, mais par Robert Laffont (pour Mahu ou le matériau) et Gallimard (pour Le Renard et la Boussole). En 1956, c'est Minuit qui publie Graal flibuste. La même année, Mahu rejoint le catalogue. Ce sera le tour du Renard et la 
Boussole en 1971. Le trajet de Claude Simon est encore différent. Quand il entre aux Éditions de Minuit, en 1957 avec Le Vent, Simon est déjà l'auteur de quatre romans : Le Tricheur et La Corde raide aux Éditions du Sagittaire, Gulliver et Le Sacre du printemps chez Calmann-Lévy. En même temps, il n'est pas inconnu chez Minuit : Lindon a racheté le catalogue du Sagittaire en 1951 - avant de le revendre rapidement (voir Simonin, 1994 : 428-434). Le contrat de Simon au Sagittaire est cédé par Pascal Pia en 1957 (Simonin, 1994 : 429). Mais Simon reniera lui-même ces premiers ouvrages.

Ce qui nous importe ici, dans ce travail de republication sous la couverture étoilée des Éditions de Minuit - donc de réappropriation -, c'est l'unité qui est créée à la réception.

17.. Les ouvrages de Simon parus chez Skira ou Maeght, par exemple, ne sont pas des romans, mais des ouvrages d'art (photographies, dessins et commentaires).

18.. Première publication en 1939 chez Denoël ; les droits sont rachetés par Lindon ; l'édition « Minuit » est une édition augmentée.

19.. On peut se référer par exemple aux chiffres de vente des romans de Beckett la première année de leur parution : 694 exemplaires pour Molloy, 241 pour Malone meurt, 476 pour L'Innommable (avec réserves ; voir Simonin, 1994 : 379).

20.. En dehors de conférences ou d'entretiens, ou, pour Simon, du Discours de Stockholm, prononcé en 1985, à l'occasion de la remise du prix Nobel de littérature.

21.. C'est le programme que développeront Philippe \& Piat (à paraître). En attendant, on peut se reporter à Philippe (2002).

22.. Sur l'ensemble de cette question, voir Philippe (2006).

23.. C'est par exemple l'horizon de Bruneau (1948-1953).

24.. Voir Piat (2005).

25.. Et qu'il est donc impossible de citer ici.

26.. C'est-à-dire d' " éléments non nécessaires à [1]a cohésion grammaticale » de l'unité textuelle (Gardes-Tamine, $2004: 89$ ).

27.. Voir, par exemple, la réflexion de Fontvieille sur cette question, et notamment, pour Simon, l'intuition d'un « nouveau modèle de phrase » (2005: 124).

28.. Authier-Revuz (1995, t. I : II)

29.. Nous soulignons, comme dans les exemples suivants.

30.. Les configurations méta-énonciatives ne sont qu'un des possibles du patron " génétique », qui permet de décrire l'existence d'une formulation première, n'ayant fait l'objet d'aucune mise en forme a posteriori dans la phrase. Outre les commentaires méta-énonciatifs, pourraient être évoqués la syntaxe hyperbatique (un élément ajouté in extremis apparaît à droite, sans avoir provoqué le remaniement de la structure phrastique), la gestion minimale de l'intégration propositionnelle et informationnelle (multiplication des incidentes, cohésion faible, changement thématique brusque, etc.), le non-effacement des marques d'hésitation et des formulations non retenues, etc.

31.. Le patron génétique peut en effet se combiner avec le patron maximaliste, lorsque le commentaire est inséré dans la phrase. Il peut aussi participer du morcellement phrastique.

32. Voir notamment Authier-Revuz (1995, I : 126-127).

33.. Cité par Bikialo (2003 : 306) ; le soulignement n'est pas repris à l'identique.

34.. Parce qu'ils peuvent être considérés comme emblématiques des grands courants de l'époque : littérature considérée comme de gauche pour Sartre - idéologique et humaniste - de droite pour Giono - légèrement gratuite, emportée par un mouvement romanesque proprement esthétique - et les Hussards - qui réagissent explicitement à la littérature engagée. 
Or, les conséquences de ces appartenances ont pu être commentées en termes stylistiques. Dans une interview collective des Hussards, intitulée «Existe-t-il un style littéraire de droite ?» ( $a$ Parisienne, $n^{\circ} 29$, juin 1955), Audiberti définit ce style comme une prose volontiers négligée, très fluide, désinvolte, etc.; le style littéraire de gauche est, lui, habité par « une sorte de hantise matérielle et carrée de la phrase ». Dans ce cas, on peut envisager le Nouveau Roman comme une troisième voie.

35.. Voir Philippe (2002).

36. Ce qui est aussi un moyen de réévaluer pour le valider linguistiquement ce que la critique littéraire a pu dire de la poétique du Nouveau Roman - autour de cette trop fameuse « aventure d'une écriture », évoquée par Ricardou, et qui a si souvent condamné la réflexion à demeurer superficielle.

37.. Voir Piat \& Philippe (2006).

38. Voir à ce sujet Philippe (à paraître). L'impression de gauchissement stylistique serait, en fait, paradoxalement créée par la reprise décalée de tours attestés en français classique.

\section{RÉSUMÉS}

Vers une stylistique des imaginaires langagiers

Si la description du matériau langagier mis en jeu dans tel ou tel corpus se situe à la base de toute analyse stylistique, la visée ultime de l'opération est d'approcher un style, catégorie littéraire éminemment polysémique. L'analyse stylistique, en effet, peut avoir pour finalité de dégager les habitudes langagières de tel auteur; elle peut aussi vouloir évaluer cette pratique en l'historicisant - ne serait-ce que parce que depuis le milieu du XIX ${ }^{\mathrm{e}}$ siècle, la langue littéraire présente des formes particulières, et que la conception du style, dès lors, s'en trouve modifiée. C'est là le postulat méthodologique dont on partira. Le corollaire d'une telle perspective est qu'une œuvre, quelle qu'elle soit, reflète un imaginaire langagier inscrit dans tel ou tel «moment » de l'histoire littéraire. La démarche ne peut être que comparative ; elle suppose donc la constitution d'un corpus pluriel dont la pertinence est définie par une proximité d'effets, qui reposent eux-mêmes sur la récurrence de "patrons " stylistiques. Mais afin de ne pas fausser le jeu, il faut nécessairement solliciter des corpus périphériques (et on se demandera comment les définir), permettant de mettre en évidence des ressemblances et des filiations aussi bien que des divergences: ce n'est qu'ainsi que l'on rendra compte de la complexité mais aussi de l'organisation du champ littéraire à un moment donné. C'est cette méthode d'analyse stylistique que l'on essaie de mettre en œuvre à partir des romans de Samuel Beckett, Robert Pinget et Claude Simon parus dans les années 1950.

Stylistics and Imaginary of Language

Through the description of the linguistic tools at stake in some corpus, stylistic analysis always aims at putting into relief a specific style - the definition of which is always to be built. Stylistic analysis may focus on one author's usual patterns as well as it may be used to evaluate such a praxis in relation with a broader context - be it internal, regarding the history of literary language, or external, in terms of position within the literary field. That is mostly true for post-1850 autonomized literature, literature having been ever since a question of language. This latter method - which will be ours in this study -implies that a literary work not only reflects 
one's imaginary of language, but enables to situate it within the dominant imaginary of language for such-and-such "moment" in literary history. To confront such problems, comparisons are required, which leads to consider a plural corpus united yet through common effects, based on identifiable and recurrent stylistic patterns. Other comparisons entail what we would call sidecorpuses (we will also discuss how they should be built), making differences and similarities obvious. This is the way one can understand both the complexity and the organisation of the literary field at one given moment. We will test these hypotheses through Beckett, Pinget and Simon's 1950s works.

\section{INDEX}

Mots-clés : stylistique, corpus, patrons stylistiques, contexte, minimalisme syntaxique, maximalisme syntaxique, patron génétique, corpus historicisé, corpus périphériques, imaginaires langagiers, Nouveau Roman, Samuel Beckett, Robert Pinget, Claude Simon

\section{AUTEUR}

JULIEN PIAT

Université Stendhal-Grenoble 3 